\title{
Gestational diabetes mellitus - literature review on selected cytokines and hormones of confirmed or possible role in its pathogenesis
}

\author{
Joanna Świrska ${ }^{1,2}$, Agnieszka Zwolak ${ }^{1,2}$, Marta Dudzińska ${ }^{1}$, Beata Matyjaszek-Matuszek², \\ Tomasz Paszkowski ${ }^{3}$ \\ ${ }^{1}$ Chair of Internal Medicine and Department of Internal Medicine in Nursing, Medical University in Lublin, Poland \\ ${ }^{2}$ Department of Endocrinology, Medical University in Lublin, Poland \\ ${ }^{3} 3$ rd Chair and Department of Gynecology, Medical University in Lublin, Poland
}

\begin{abstract}
:
The incidence of gestational diabetes mellitus (GDM) increases globally, including Poland. Considering serious consequences of gestational diabetes for both mother and fetus, screening for this disorder is an obligatory element of managing pregnant woman. The pathogenesis of gestational diabetes is not yet thoroughly explained. However, it is insulin resistance and chronic subclinical inflammatory process which are considered to be major factors responsible for the development of GDM. These two states are triggered mainly by secretion of proinflammatory cytokines and by abnormal function of adipose tissue.

The study reviews the literature on selected hormones and cytokines whose role in the GDM pathogenesis has been already confirmed as well as on those proteins whose role is either not yet fully understood or which may possibly participate in GDM development. Owing to the fact that underlying mechanisms of GDM are, in general, similar to the mechanisms responsible for metabolic disorders such as diabetes mellitus type 2 or obesity, in this review we focus first on the role these molecules play in pathogenesis of metabolic disorders and then present current state of knowledge on their action in gestational diabetes development. The review presents: TNF alpha, adipokines - adiponectin and leptin and relatively newly discovered proteins: fetuin $A$, periostin, angiopoietin-like protein 8 or high mobility group box.
\end{abstract}

Key words: gestational diabetes, metabolic disorders, cytokines, hormones

Ginekologia Polska 2018; 89, 9: 522-527

\section{INTRODUCTION}

Gestational diabetes mellitus (GDM) is a glucose intolerance disorder with onset or first recognition during pregnancy, which, however, does not meet the criteria of diabetes mellitus for the general population [1, 2]. Based upon available publications, the prevalence of GDM varies widely - from $1 \%$ to $22 \%$ of pregnant women, depending on the examined group of patients and adopted GDM diagnostic criteria. Its frequency increases globally [1-8]. Screening for GDM is an obligatory element of managing pregnant woman in many countries, including Poland. The reason for this is that GDM is related to several short and long-term complications.
Of special concern is the fact that, in the long term, there is a higher risk of developing diabetes mellitus type 2 in the future in both mother affected by GDM and in her offspring. In the woman with a history of GDM, the incidence of DM2 is estimated from $3 \%$ to even over $90 \%$, depending mainly on the study design and observation time. This is true for both lean and obese women.

Considering the growing incidence of GDM and the complications related to this, it is not surprising that big efforts are made to fully understand its pathogenesis. Understanding GDM background could facilitate predicting its development in pregnant women and lead to the introduction of new treatment methods. Thus, the aim of this study 
was to review the literature on selected hormones and cytokines - on those whose role in the GDM pathogenesis has been already confirmed as well as on those whose role is either not yet fully understood or which may possibly participate in GDM development. Owing to the fact that underlying mechanisms of GDM are in general, similar to the mechanisms responsible for metabolic disorders such as DM2 or obesity (see below), in this review we focus first on the role of these molecules in pathogenesis of metabolic disorders and then present current state of knowledge on their action in GDM development.

\section{Pathogenesis of gestational diabetes mellitus - general information}

Although pathogenesis of GDM is not yet thoroughly explained, it is insulin resistance (IR) and chronic subclinical inflammatory process which are considered major factors responsible for its development. They are also the key pathogenetic mechanisms of obesity and other metabolic disorders, including DM2, as mentioned above. Inflammation and IR are triggered by abnormal activity of adipose tissue. The latter is a source of inflammatory mediators. It is also a place of interaction between adypocytes and immunocompetent cells. The studies on animal model and in humans demonstrate that adypocytes of obese individuals secrete proinflammatory cytokines, which, in turn, enhance infiltration of macrophages in adipose tissue. Furthermore, adipose tissue secretes adipokines, which also play a vital role in the development of IR and metabolic disorders such as DM2 or GDM [9].

\section{Tumor necrosis factor alpha — proinflammatory cytokine}

Tumor necrosis factor alpha (TNF alpha) is a proinflammatory cytokine, which has a major role in the development of IR and DM2 [10]. It is produced by monocytes and macrophages in the adipose tissue [11]. It has proapoptotic activity, affects cell differentiation and recruitment. Acting via tumor necrosis factor receptor 1 (TNFR-1), it activates immunocompetent cells to spread inflammatory process. It also induces oxidative stress. Oxidative stress alongside inflammation promote cells degeneration $[10,12]$. Furthermore, TNF alpha stimulates prostaglandins synthesis by inducing cyclooxygenase 2 . By activating several caspases, it enhances apoptosis of the inflammed cells [13]. The studies demonstrate excessive expression of TNF alpha in the adipose tissue in obese individuals compared to lean subjects [14]. TNF alpha promotes IR by suppressing tyrosin phosphorylation of insulin receptor and its substrate-insulin receptor substrate-1 (IRS-1) because it inhibits action of tyrosine kinase [15]. Furthermore, it diminishes expression of glucose transporter type 4 (GLUT 4) in the adipose tissue, skeletal muscles and cardiac muscle. Proinflamamtory activ- ity of TNF alpha in beta cells of the pancreas plays a vital role in their apoptosis, contributing thereby to diabetes mellitus development [10]. Administration of TNF-alpha antagonist etanercept in obese human subjects with metabolic syndrome decreased fasting glucose in 6-month observation time but did not change insulin sensitivity [16].

Based upon the available literature, more TNF alpha is secreted in pregnancy, particularly in third trimester. This is true for both normal pregnancy and for the pregnancy complicated by GDM $[17,18]$. Highest levels of TNF alpha are found in GDM. Xu performed a retrospective meta-analysis of 27 trials where concentration of TNF alpha in pregnancy was evaluated. Significantly higher TNF alpha values were found in women with GDM when compared to pregnant women with normoglycemia. In the studies, the differences in TNF alpha concentrations were significant also after ruling out the influence of the body mass [4].

In pregnancy, TNF alpha is secreted not only by adipose tissue but also by placenta. It seems that excessive amount of TNF alpha in GDM originates from placental secretion rather than from adipose tissue production. According to Kirwan, gestational insulin resistance and impaired glucose tolerance are mainly due to elevated TNF alpha (and to less extent to higher leptin and cortisol levels), rather than to excessive secretion of placental hormones such as prolactin, human chorionic gonadotropin (hCG) or human placental lactogen (HPL) as it was previously thought [19].

\section{Adipokines - leptin and adiponectin}

Adipokines are hormones secreted by adipose tissue; their impaired secretion is related to several metabolic disorders such as insulin resistance, obesity, DM2, cardiovascular diseases or GDM.

Of these, leptin has been examined best to this day. Apart from the role in the metabolic diseases development, it plays a role in the puberty and in angiogenesis [20]. Leptin is called a"satiety hormone" because it regulates food intake and energy expenditure. It acts via leptin receptors which are found in the hypothalamus [1, 21]. Its secretion is regulated, among others, by insulin and cortisol concentrations [22]. A reverse correlation was found between leptin and insulin sensitivity. In obese individuals, leptin's concentration is elevated and its exogenous supplementation does not lead to body mass reduction, thus suggesting resistance to this hormone in fat individuals. At the same time, body mass reduction results in leptin blood level decline and insulin sensitivity increase [23]. It is postulated that hyperleptinemia suppresses insulin-dependent glucose transport to adipocytes [1, 24]. Furthermore, it may directly impair glucose secretion by beta cells of the pancreas $[1,25]$. Obese individuals without insulin resistance have lower leptin levels compared to fat subjects in whom insulin resistance is present [26]. 
In physiologic pregnancy as well as in the pregnancy complicated by GDM, leptin's concentration increases gradually with the highest values detected in 28th week of gestation and is followed by plateau, to decrease eventually before delivery [22]. In pregnancy, leptin is secreted not only by adipose tissue but also by placenta. Its concentration in GDM is higher than in normoglycemic pregnancy and higher than in pregnant-diabetes-mellitus-type 1 patients. However, the data on the main source of leptin in GDM - whether it is adipose tissue or placenta - are contradictory. The majority of studies prove positive correlation between leptin's concentration with body weight and body mass index (BMI). It is true for both pregnant and non-pregnant women [22, 27].

Adiponectin is an adipokine with anti-inflammatory activity. It inhibits lipopolysaccharide (LPS)-stimulated TNF-alpha production by macrophages, enhances secretion of anti-inflammatory II-10, and stimulates the action of macrophages M2. The latter are responsible for the phagocytosis of apoptotic cells. Enhancement of apoptotic cells clearance by macrophages $M 2$ protects the organism from systemic inflammation $[28,29]$. Adiponectin expression in adipose tissue is diminished by TNF alpha and II-6. This explains why adiponectin's concentration declines in the states related to subclinical inflammation such as obesity, IR or DM2 [28-30]. Adiponectin's concentration is also lower in the smokers, in cardiovascular disease and in polycystic ovary syndrome (PCOS) [31]. Besides, its low concentrations are found in selected malignant neoplasms [32].

Adiponectin sensitizes the tissues to insulin indirectly due to the anti-inflammatory action mentioned above. It also increases insulin sensitivity by stimulating glucose uptake in skeletal muscles and by diminishing hepatic gluconeogenesis. Exogenous administration of adiponectin to laboratory animals decreases IR in them [33]. Adiponectin is believed to suppress development of atherosclerosis, fatty liver disease and liver fibrosis [28, 34].

In pregnancy, adiponectin is secreted mainly by adipose tissue. It remains controversial whether placenta could be an additional source of this hormone [1, 32]. Generally, pregnancy is regarded as a state of physiologic hypoadiponectinemia. In fact, several studies proved decline of adiponectin concentration in both physiologic pregnancy and in GDM [30, 32, 35]. Williams found that low adiponectin concentration in early pregnancy is a predictor of GDM development in the third trimester regardless woman's age, pregravid obesity or familial history of DM2 [31].

\section{Angiopoietin-like protein 8}

Angiopoietin-like protein 8 (ANGPTL8), also known as lipasin or betatrophin, is a recently discovered hormone. It is produced mainly by the liver and in adipose tissue [36,
37]. It takes part in glucose and lipids homeostasis - inhibits lipoprotein lipase activity and, according to some authors, also stimulates pancreas beta cells proliferation in IR states. It is postulated that liver is the main source of this protein [38, 39]. The liver expression of ANGPTL8 is elevated in laboratory animals with IR. However, beta cells growth followed by enhanced insulin secretion due to ANGPTL8 supplementation which was found in the animals was not confirmed in humans $[39,40]$. Higher ANGPTL8 concentrations were found in obese individuals and in DM2 patients [36]. In the study by Wang, in women with PCOS and with IR, higher concentrations of ANGPTL8 were found in comparison to healthy women. Hormone's concentration correlated positively with $\mathrm{BMI}$, waist to hip ratio (WHR), triglycerides (TG), total cholesterol (TC), free androgen index (FAl), and homeostasis model assessment for insulin resistance (HOMA-IR). Furthermore, hepatocytes' stimulation by insulin (EHC, hyperinsulemic euglycemic clamp) enhanced ANGPTL8 expression, while metformin or rosiglitason therapy declined the hormone's level [41].

The majority of studies point the role of ANGPTL8 in the development of metabolic disorders. Yet, there are authors who deny these observations. For example, Gomez-Ambrosi found decreased ANGPTL8 level in DM2 and in obesity. In this study, the lowest ANGPTL8 concentrations were found in subjects where obesity was associated with IR [39].

It has been suggested that significant discrepancies in ANGPTL 8 concentrations among different studies may be due to the fact that different kits were used in these trials. Different kits measure different betatrophin species — full-length protein, C-terminal fragments or $\mathrm{N}$-terminal fragments [42]. Physiologically, full-length protein is cleaved from pro-protein, releasing $\mathrm{C}$-terminal fragment. This post-translational regulation of betatrophin concentration determines the presence of different betatrophin fragments in serum. According to $\mathrm{Fu}$, it seems that kits with C-terminal antibodies recognize both $\mathrm{C}$-fragments and full-length protein. On the other hand, ELISA kits that measure N-terminal ELISA tests, measure full-length protein only. Thus, the kits that measure C-fragments will give higher betatorophin values than the kits measuring $\mathrm{N}$-terminals. The discrepancies between the results obtained in different trials can be also due to different time of blood collection (fasting vs postprandial state). Besides, stronger expression of ANGPTL8 is caused by insulin, cold or feeding [37, 38, 42]. Weaker betarophin expression is seen in starving and in other states of enhanced lipolysis [37].

The studies point to elevated betatrophin values in pregnancy $[38,40]$. In the study by Perez, ANGPTL8 concentration in normal pregnancy was approximately ten times higher than in non-pregnant women [38]. Trebotic found significantly higher hormone concentrations in pregnancy with the highest values in pregnancies complicated by GDM 
[40]. On the other hand, Martinez-Perez also confirmed higher betatrophin values in pregnancy but its values did not differ significantly between normoglycemic pregnancy and pregnancy complicated by GDM. In the study, in all pregnant women, the highest values were found in the first trimester with a gradual decline during pregnancy. It is worth mentioning, that betatrophin concentration is higher in umbilical-cord blood than in mother's blood, suggesting its role in fetal development [38].

\section{Periostin}

Periostin is a protein which was primarily isolated from mice osteoblasts. It is found in the tissues rich in collagen such as tendons, ligaments, cardiac valves or cornea [43]. Due to its effect on mesenchymal cells, it plays a role in bone and teeth development [44]. It participates in the pathogenesis of neoplasms, arthritis, atherosclerosis or inflammatory diseases $[43,44]$. A relation was found between periostin concentration and metabolic diseases as periostin enhances c-Jun $\mathrm{N}$-terminal kinase (JNK)-dependent suppression of fatty acids oxidation in the liver. Higher glucose concentrations enhance periostin expression [44]. In the study by Luo, significantly higher periostin concentrations were found in obese patients and in patients with DM2 compared to healthy individuals. The highest concentrations were found in obese patients with concomitant DM2. Furthermore, a positive correlation was stated between periostin and TG and TC and negative - between periostin and HDL. The TG concentration was an independent factor affecting periostin level after ruling out age, sex and BMI value of the examined patients. In the study, positive correlation between periostin and TNF alpha and II-6 was found [43]. Since periostin promotes liver steatosis, it is postulated that it could serve as a marker of NAFLD as its concentrations were significantly higher in NAFLD patients compared to healthy controls $[44,45]$. Satirapoj found higher periostin concentrations in the urine of DM2 patients, even before the albuminuria onset [46].

In the available literature no articles were found on the relation between periostin concentration and GDM. Considering, however, a strong pathogenetic relation between GDM and metabolic disorders such as obesity or DM2 where periostin concentration is elevated, it can be expected that its concentration in GDM will be elevated, too.

The studies show the role of periostin in embryo implantation and development. In the study by Freis, it was suggested that periostin could serve as an early prognostic marker of miscarriage, as its rise in miscarriage is observed even before the decline in beta hCG level is detected [47].

\section{Fetuin A}

Fetuin $A$ is a multifunctional glycoprotein produced mainly in the liver $[48,49]$. It plays a role in blood-vessels cal- cification and in bone metabolism. Its higher concentrations are found in IR related to PCOS or DM2 [49]. Furthermore, a positive correlation was found between fetuin $A$ level and the risk of developing DM2 in women. The role of fetuin $A$ in metabolic disorders is due to insulin receptor inhibition by interacting in thyrosin kinase action [50]. In skeletal muscles and in the liver, a direct inhibitory effect of fetuin A on autophosphorylation of insulin receptor was detected [49]. The study on animals showed that lack of fetuin A protects them against age-related loss of insulin sensitivity [50]. It was also found that DM2 patients with higher glycated hemoglobin $(\mathrm{HbA} 1 \mathrm{C})$ values $(>=7.0 \%)$ had higher fetuin A concentrations than patients with lower $\mathrm{HbA} 1 \mathrm{C}$ levels (< $6.5 \%)$, suggesting the influence of chronic hyperglycemia on fetuin A concentration (51). However, following lydir, there are also studies which deny any relation between DM2 and fetuin A concentration [49].

The results on fetuin A concentration in normal pregnancy and in pregnancy complicated by GDM are contradictory. Kalabay showed higher fetuin A concentration in GDM. He also confirmed positive correlation between fetuin $A$ and C-peptyde level [52]. Similarly, lydir found higher fetuin A concentration in GDM when compared to healthy women. Furthermore, he stated a correlation between $\mathrm{HbA} 1 \mathrm{C}$ and fetuin A level and between fetuin A and TG or TC level [49]. On the other hand, Farhan did not show any differences in fetuin A concentration in the third trimester between normoglycemic women and women in whom in the course of OGTT GDM was detected [48].

\section{High mobility group box 1}

High mobility group box 1 (HMBG-1) is a protein which migrates quickly during electrophoresis and thus was named for this feature. It is a non-histone protein responsible for the maintenance of nucleosome structure in the cell nucleus. It takes part in the regulation of genes' transcription and in the repairing of chromosomal DNA [53, 54]. HMBG-1 is released from damaged cells, it can be also secreted to the extracellular space after cell activation by immunocompetent cells. Monocytes and macrophages, stimulated by cytokines such as IFN-y, TNF alpha or II-1, secrete actively HMBG-1 [54].

It plays a role in inflammations - both infectious and non-infectious. For example, it promotes the development of septic shock, systemic lupus erythematosus, disseminated intravascular coagulation, cardiovascular diseases or it is involved in the endothelium dysfunction. The studies confirm that HMBG-1 participates in IR development [53]. Arrigo showed extremely high HMBG-1 values in obese children, and its concentration was in the positive correlation with other proinflammatory cytokines such as TNF alpha, II-6, II-18 [55]. 
A positive correlation was found in pregnant women between HMBG-1 concentration and HOMA-IR, glucose concentrations in OGTT and with pre-gravid BMI value. HMBG-1 was an independent risk factor of GDM [53]. The fact that HMBG-1 is a marker of inflammatory process and that it is elevated in GDM, indirectly confirms the role of inflammation in GDM development.

\section{CONCLUSION}

Considering the growing incidence of GDM and the consequences related to this, it is necessary to fully understand its pathogenesis - the studies on relatively newly discovered proteins such as fetuin A, periostin, angiopoietin-like protein 8 or high mobility group box 1 may provide new insights into this matter.

\section{REFERENCES}

1. Wójcik M, Chmielewska-Kassassir M, Grzywnowicz K, et al. The relationship between adipose tissue-derived hormones and gestational diabetes mellitus (GDM). Endokrynol Pol. 2014; 65(2): 134-142, doi: 10.5603/EP.2014.0019, indexed in Pubmed: 24802737.

2. Bener A, Saleh NM, Al Hamaq A. 640 Prevalence of Gestational Diabetes and Associated Maternal and Neonatal Complications in a Fast Developing Community: Global Comparisons in a fast developing community: global comparison. Int J Womens Health. 2011; 3: 367-373.

3. Atègbo JM, Grissa $\mathrm{O}$, Yessoufou $\mathrm{A}$, et al. Modulation of adipokines and cytokines in gestational diabetes and macrosomia. J Clin Endocrinol Metab. 2006; 91(10): 4137-4143, doi: 10.1210/jc.2006-0980, indexed in Pubmed: 16849405.

4. Xu J, Zhao Y, Chen Y, et al. Maternal Circulating Concentrations of Tumor Necrosis Factor-Alpha, Leptin, and Adiponectin in Gestational Diabetes Mellitus: A Systematic Review and Meta-Analysis. The Scientific World Journal. 2014; 2014: 1-12, doi: 10.1155/2014/926932.

5. Melchior $H$, Kurch-Bek D, Mund M. The Prevalence of Gestational Diabetes. Dtsch Arztebl Int. 2017; 114(24): 412-418, doi: 10.3238/arztebl.2017.0412, indexed in Pubmed: 28669379.

6. Wielgoś M, Bomba-Opoń D, Czajkowski K, et al. Towards a European Consensus on Gestational Diabetes Mellitus: A Pragmatic Guide for Diagnosis, Management, and Care. The Polish Diabetes in Pregnancy Study Group and FIGO. Ginekologia Polska. 2017; 88(1): 46-49, doi: 10.5603/gp.a2017.0010.

7. Metzger BE. Long-term outcomes in mothers diagnosed with gestational diabetes mellitus and their offspring. Clin Obstet Gynecol. 2007; 50(4): 972-979, doi: 10.1097/GRF.0b013e31815a61d6, indexed in Pubmed: 17982340.

8. Löbner K, Knopff A, Baumgarten A, et al. Predictors of postpartum diabetes in women with gestational diabetes mellitus. Diabetes. 2006; 55(3): 792-797, indexed in Pubmed: 16505245.

9. Hotamisligil GS. Inflammation and metabolic disorders. Nature. 2006; 444(7121): 860-867, doi: 10.1038/nature05485, indexed in Pubmed: 17167474.

10. Akash MS, Rehman K, Liaqat A. Tumor Necrosis Factor-Alpha: Role in Development of Insulin Resistance and Pathogenesis of Type 2 Diabetes Mellitus. J Cell Biochem. 2018; 119(1): 105-110, doi: 10.1002/jcb.26174, indexed in Pubmed: 28569437.

11. Gao L, Gu Y, Yin X. High Serum Tumor Necrosis Factor-Alpha Levels in Women with Polycystic Ovary Syndrome: A Meta-Analysis. PLoS One. 2016; 11(10): e0164021, doi: 10.1371/journal.pone.0164021, indexed in Pubmed: 27764100.

12. Fischer $R$, Maier $O$. Interrelation of oxidative stress and inflammation in neurodegenerative disease: role of TNF. Oxid Med Cell Longev. 2015; 2015: 610813, doi: 10.1155/2015/610813, indexed in Pubmed: 25834699.

13. Sprague $A H$, Khalil RA. Inflammatory cytokines in vascular dysfunction and vascular disease. Biochem Pharmacol. 2009; 78(6): 539-552, doi: 10.1016/j.bcp.2009.04.029, indexed in Pubmed: 19413999.

14. Hotamisligil GS, Arner P, Caro JF, et al. Increased adipose tissue expression of tumor necrosis factor-alpha in human obesity and insulin resistance.
J Clin Invest. 1995; 95(5): 2409-2415, doi: 10.1172/JCI117936, indexed in Pubmed: 7738205.

15. Hotamisligil GS, Budavari A, Murray D, et al. Reduced tyrosine kinase activity of the insulin receptor in obesity-diabetes. Central role of tumor necrosis factor-alpha. J Clin Invest. 1994; 94(4): 1543-1549, doi: 10.1172/JCl117495, indexed in Pubmed: 7523453.

16. Stanley TL, Zanni MV, Johnsen S, et al. TNF-alpha antagonism with etanercept decreases glucose and increases the proportion of high molecular weight adiponectin in obese subjects with features of the metabolic syndrome. J Clin Endocrinol Metab. 2011; 96(1): E146-E150, doi: 10.1210/jc.2010-1170, indexed in Pubmed: 21047923.

17. Cseh K, Baranyi E, Melczer Z, et al. Tumor necrosis factor system in insulin resistance in gestational diabetes. Diabetes Res Clin Pract. 2002; 56(2): 93-99, indexed in Pubmed: 11891016.

18. Barbour LA, McCurdy CE, Hernandez TL, et al. Cellular mechanisms for insulin resistance in normal pregnancy and gestational diabetes. Diabetes Care. 2007; 30 Suppl 2: S112-S119, doi: 10.2337/dc07-s202, indexed in Pubmed: 17596458.

19. Kirwan JP, Mouzon SHD, Lepercq J, et al. TNF- Is a Predictor of Insulin Resistance in Human Pregnancy. Diabetes. 2002; 51(7): 2207-2213, doi: 10.2337/diabetes.51.7.2207.

20. Al-Badri MR, Zantout MS, Azar ST. The role of adipokines in gestational diabetes mellitus. Ther Adv Endocrinol Metab. 2015; 6(3): 103-108, doi: 10.1177/2042018815577039, indexed in Pubmed: 26137214.

21. Osegbe I, Okpara H, Azinge E. Relationship between serum leptin and insulin resistance among obese Nigerian women. Ann Afr Med. 2016; 15(1): 14-19, indexed in Pubmed: 26857932.

22. Kautzky-Willer A, Pacini G, Tura A, et al. Increased plasma leptin in gestational diabetes. Diabetologia. 2001; 44(2): 164-172, doi: 10.1007/s001250051595, indexed in Pubmed: 11270672.

23. Wang TN, Chang WT, Chiu YW, et al. Relationships between changes in leptin and insulin resistance levels in obese individuals following weight loss. Kaohsiung J Med Sci. 2013; 29(8): 436-443, doi: 10.1016/j. kjms.2012.08.041, indexed in Pubmed: 23906234.

24. Pérez C, Fernández-Galaz C, Fernández-Agulló T, et al. Leptin impairs insulin signaling in rat adipocytes. Diabetes. 2004; 53(2): 347-353, indexed in Pubmed: 14747284.

25. Marroquí $L$, Gonzalez A, Neco $P$, et al. Role of leptin in the pancreatic $\beta$-cell: effects and signaling pathways. J Mol Endocrinol. 2012; 49(1): 164-172, doi: 10.1530/JME-12-0025, indexed in Pubmed: 22448029.

26. Zuo H, Shi Z, Yuan B, et al. Association between serum leptin concentrations and insulin resistance: a population-based study from China. PLoS One. 2013; 8(1): 1-7, doi: 10.1371/journal.pone.0054615, indexed in Pubmed: 23349940.

27. Sattar N, Greer IA, Pirwani I, et al. Leptin levels in pregnancy: marker for fat accumulation and mobilization? Acta Obstet Gynecol Scand. 1998; 77(3): 278-283, indexed in Pubmed: 9539272.

28. Kwon H, Pessin J. Adipokines Mediate Inflammation and Insulin Resistance. Frontiers in Endocrinology. 2013; 4, doi: 10.3389/fendo.2013.00071.

29. Takemura $Y$, Ouchi $N$, Shibata $R$, et al. Adiponectin modulates inflammatory reactions via calreticulin receptor-dependent clearance of early apoptotic bodies. J Clin Invest. 2007; 117(2): 375-386, doi: 10.1172/JCl29709, indexed in Pubmed: 17256056.

30. Catalano PM, Hoegh M, Minium J, et al. Adiponectin in human pregnancy: implications for regulation of glucose and lipid metabolism. Diabetologia. 2006; 49(7): 1677-1685, doi: 10.1007/s00125-006-0264-x, indexed in Pubmed: 16752186.

31. Williams MA, Qiu C, Muy-Rivera M, et al. Plasma adiponectin concentrations in early pregnancy and subsequent risk of gestational diabetes mellitus. J Clin Endocrinol Metab. 2004; 89(5): 2306-2311, doi: 10.1210/jc.2003-031201, indexed in Pubmed: 15126557.

32. Haghiac M, Basu S, Presley L, et al. Patterns of adiponectin expression in term pregnancy: impact of obesity. J Clin Endocrinol Metab. 2014; 99(9): 3427-3434, doi: 10.1210/jc.2013-4074, indexed in Pubmed: 24796925.

33. Vrachnis N, Belitsos P, Sifakis S, et al. Role of Adipokines and Other Inflammatory Mediators in Gestational Diabetes Mellitus and Previous Gestational Diabetes Mellitus. International Journal of Endocrinology. 2012; 2012: 1-12, doi: 10.1155/2012/549748.

34. Okamoto Y. Adiponectin Provides Cardiovascular Protection in Metabolic Syndrome. Cardiology Research and Practice. 2011; 2011: 1-7, doi: 10.4061/2011/313179.

35. Noureldeen AFH, Qusti SY, Al-Seeni MN, et al. Maternal leptin, adiponectin, resistin, visfatin and tumor necrosis factor-alpha in normal and 
gestational diabetes. Indian J Clin Biochem. 2014; 29(4): 462-470, doi: 10.1007/s12291-013-0394-0, indexed in Pubmed: 25298627.

36. Wang H, LaiY, Han C, et al. The Effects of Serum ANGPTL8/betatrophin on the Risk of Developing the Metabolic Syndrome - A Prospective Study. Scientific Reports. 2016; 6(1), doi: 10.1038/srep28431.

37. Quagliarini F, Wang Y, Kozlitina J, et al. Atypical angiopoietin-like protein that regulates ANGPTL3. Proc Natl Acad Sci U S A. 2012; 109(48): 19751-19756, doi: 10.1073/pnas.1217552109, indexed in Pubmed: 23150577.

38. Martinez-Perez B, Ejarque M, Gutierrez C, et al. Angiopoietin-like protein 8 (ANGPTL8) in pregnancy: a brown adipose tissue-derived endocrine factor with a potential role in fetal growth. Translational Research. 2016; 178: 1-12, doi: 10.1016/j.trsl.2016.06.012.

39. Gómez-Ambrosi J, Pascual E, Catalán V, et al. Circulating betatrophin concentrations are decreased in human obesity and type 2 diabetes. J Clin Endocrinol Metab. 2014; 99(10): E2004-E2009, doi: 10.1210/jc.20141568, indexed in Pubmed: 25050901.

40. Trebotic L, Klimek P, Thomas A, et al. Circulating Betatrophin Is Strongly Increased in Pregnancy and Gestational Diabetes Mellitus. PLOS ONE. 2015; 10(9): e0136701, doi: 10.1371/journal.pone.0136701.

41. Wang H, Du L, WuT, et al. Circulating betatrophin is associated with insulin resistance in humans: cross-sectional and interventional studies and. Oncotarget. 2017; 8(57): 96604-96614, doi: 10.18632/oncotarget.21852, indexed in Pubmed: 29228555.

42. Fu Z, Abou-Samra AB, Zhang R. An explanation for recent discrepancies in levels of human circulating betatrophin. Diabetologia. 2014; 57(10): 2232-2234, doi: 10.1007/s00125-014-3346-1, indexed in Pubmed: 25099942.

43. Luo $\mathrm{Y}, \mathrm{Qu} \mathrm{H}$, Wang $\mathrm{H}$, et al. Plasma Periostin Levels Are Increased in Chinese Subjects with Obesity and Type 2 Diabetes and Are Positively Correlated with Glucose and Lipid Parameters. Mediators of Inflammation. 2016; 2016: 1-6, doi: 10.1155/2016/6423637.

44. LuY, Liu X, Jiao Y, et al. Periostin promotes liver steatosis and hypertriglyceridemia through downregulation of PPARa. J Clin Invest. 2014; 124(8): 3501-3513, doi: 10.1172/JCI74438, indexed in Pubmed: 25003192.

45. Zhu JZ, Zhu HT, Dai YN, et al. Serum periostin is a potential biomarker for non-alcoholic fatty liver disease: a case-control study. Endocrine. 2016; 51(1): 91-100, doi: 10.1007/s12020-015-0735-2, indexed in Pubmed: 26362060.
46. Satirapoj B, Tassanasorn S, Charoenpitakchai M, et al. Periostin as a tissue and urinary biomarker of renal injury in type 2 diabetes mellitus. PLoS One. 2015; 10(4): e0124055, doi: 10.1371/journal.pone.0124055, indexed in Pubmed: 25884625.

47. Freis A, Schlegel J, Kuon RJ, et al. Serum periostin levels in early in pregnancy are significantly altered in women with miscarriage. Reproductive Biology and Endocrinology. 2017; 15(1), doi: 10.1186/s12958017-0307-9.

48. Farhan S, Handisurya A, Todoric J, et al. Fetuin-A Characteristics during and after Pregnancy: Result from a Case Control Pilot Study. International Journal of Endocrinology. 2012; 2012: 1-5, doi: 10.1155/2012/896736.

49. Iyidir OT, Degertekin CK, Yilmaz BA, et al. Serum levels of fetuin A are increased in women with gestational diabetes mellitus. Arch Gynecol Obstet. 2015; 291(4): 933-937, doi: 10.1007/s00404-014-3490-3, indexed in Pubmed: 25260988.

50. Sun Qi, Cornelis MC, Manson JE, et al. Plasma levels of fetuin-A and hepatic enzymes and risk of type 2 diabetes in women in the U.S. Diabetes. 2013; 62(1): 49-55, doi: 10.2337/db12-0372, indexed in Pubmed: 22923470.

51. Liu Yu, Xu M, Xu Yu, et al. Positive correlation between chronic hyperglycemia and serum fetuin-A levels in middle-aged and elderly Chinese. J Diabetes. 2012; 4(4):351-358, doi: 10.1111/1753-0407.12000, indexed in Pubmed: 23190703.

52. Kalabay L, Cseh K, Pajor A, et al. Correlation of maternal serum fetuin/alpha2-HS-glycoprotein concentration with maternal insulin resistance and anthropometric parameters of neonates in normal pregnancy and gestational diabetes. Eur J Endocrinol. 2002; 147(2): 243-248, indexed in Pubmed: 12153747.

53. Giacobbe A, Granese R, Grasso R, et al. Association between maternal serum high mobility group box 1 levels and pregnancy complicated by gestational diabetes mellitus. Nutr Metab Cardiovasc Dis. 2016; 26(5):414418, doi: 10.1016/j.numecd.2016.02.007, indexed in Pubmed: 27089978.

54. Bertheloot D, Latz E. HMGB1, IL-1a, IL-33 and S100 proteins: dual-function alarmins. Cell Mol Immunol. 2017; 14(1): 43-64, doi: 10.1038/cmi.2016.34, indexed in Pubmed: 27569562.

55. Arrigo T, Chirico V, Salpietro V, et al. High-mobility group protein B1: a new biomarker of metabolic syndrome in obese children. Eur J Endocrinol. 2013; 168(4): 631-638, doi: 10.1530/EJE-13-0037, indexed in Pubmed: 23384711. 